\title{
Avaliação hormonal de atletas: sangue ou saliva?
}

- O monitoramento de atletas necessita coletas de sangue ao longo da temporada de treinos e competições. As avaliações têm por objetivo prevenir lesões ou diagnosticar patologias que prejudicam o desempenho;

- A saliva é um fluído biológico menos invasivo, de fácil obtenção e menos estressante para avaliar o atleta sem interferir em sua rotina;

- A melhor escolha para coleta e análise de saliva é o método passivo e sem estimulação artificial;

- A concentração salivar de testosterona e cortisol apresenta correlação significativa com os valores de testosterona e cortisol livre no plasma;

- A avaliação de testosterona salivar fornece resultados mais confiáveis em homens e menor sensibilidade em mulheres;

- A concentração de cortisol, DHEA, testosterona e androstenediona em saliva podem variar de acordo com o ciclo circadiano, apresentando valores mais elevados pela manhã;

- A dosagem de cortisol salivar antes e imediatamente após o exercício, pode auxiliar o nutricionista na estratégia mais adequada de recuperação do atleta;

- A enzima alfa amilase salivar pode ser utilizada como um biomarcador de estresse físico e psicológico em atletas e pessoas fisicamente ativas;

- A utilização de dispositivos de coleta de saliva a base de algodão ou polipropileno, são adequados apenas para a dosagem de cortisol e interferem na analise de testosterona livre;

- A análise da relação testosterona/androstenediona na saliva pode ser uma ferramenta diagnóstica interessante para avaliar portadoras da síndrome dos ovários policísticos.

\section{Referências bibliográficas}

1.CLIFTON, S.; MACDOWALL, W.; COPAS, A.J. et al. Salivary testosterone levels and health status in men and women in the British general population: Findings from the third national survey of sexual attitudes and lifestyles (Natsal-3). J Clin Endocrinol Metab; 101(11):3939-51, 2016. 2. LEWIS JG. Steroid analysis in saliva: an overview. Clin Biochem Rev; 27(3):139-46, 2006.

3. KEEVIL, B.G.; CLIFTON, S.; TANTON, C. et al. Distribution of Salivary Testosterone in Men and Women in a British General Population-Based Sample: The Third National Survey of Sexual Attitudes and Lifestyles (Natsal-3). J Endocr Soc; 1:14-25, 2017.

4. MÜNZKER, J.; LINDHEIM, L.; ADAWAY, J. et al. High salivary testosterone-to-androstenedione ratio and adverse metabolic phenotypes in women with polycystic ovary syndrome. Clin Endocrinol (Oxf); 86(4):567-75, 2017.

5. NUNES, L.A.S.; MUSSAVIRA, S.; BINDHU, O.S. Clinical and diagnostic utility of saliva as a non-invasive diagnostic fluid: A systematic review. Biochem Medica; 25(2): 177-92, 2015. 